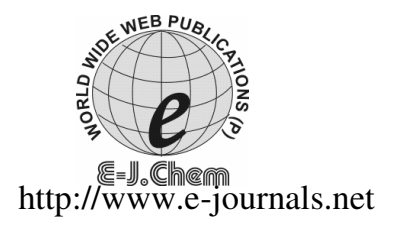

ISSN: 0973-4945; CODEN ECJHAO

E-Journal of Chemistry

2010, 7(4), 1230-1237

\title{
A Comparative Study of Inhibitive Effects of Some Schiff Bases on Mild Steel in Acidic Media by Mass Loss Techniques
}

\author{
ABHA GOYAL, V.K. SWAMI and S.P. MATHUR* \\ Research Laboratory, \\ Government Lohia (P.G.) College, Churu-331001, India. \\ abhag_chem@yahoo.co.in
}

Received 8 December 2009; Accepted 5 February 2010

\begin{abstract}
Mass loss techniques have been employed to study the corrosion inhibition of some newly synthesized Schiff bases. viz. N-4-(Diethyl amino) salicylidine-2-amino-5-chloropyridine $\left(\mathrm{SB}_{1}\right), N$-(4-ethylbenzalidine)-2-amino-5chloropyridine $\left(\mathrm{SB}_{2}\right), N$-(4-ethylbenzalidine)-2-amino-5-bromopyridine $\left(\mathrm{SB}_{3}\right)$, $N$-4-(Diethyl amino) salicylidine-2-amino-5-bromopyridine $\left(\mathrm{SB}_{4}\right)$ for mild steel in $\mathrm{HCl}$ and $\mathrm{H}_{2} \mathrm{SO}_{4}$ solutions. Results of inhibition efficiencies from the mass loss technique show that Schiff bases are good inhibitors in both the acidic solutions. Inhibition efficiencies increase with the increase in the concentration of acids as well as those of inhibitors. Maximum efficiency for $\mathrm{H}_{2} \mathrm{SO}_{4}$ was found $99.78 \%$ and for $\mathrm{HCl}$ it was $97.92 \%$.
\end{abstract}

Keywords: Corrosion, Schiff Bases, Corrosion rate, Surface coverage.

\section{Introduction}

Mild Steel is an important metal regarding to its wide applications in industry in various mechanical and structural purposes, like in bridgework, industrial parts, steam engine parts, automobiles, for ship hulls and off shore drilling platforms etc. Mild steel is passive towards an alkali but it is prone to corrosion in acids like $\mathrm{HCl}$ and $\mathrm{H}_{2} \mathrm{SO}_{4}$ solutions. These acids are generally used for drilling operations, Pickling baths and in descaling processes ${ }^{1}$.

Corrosion of mild steel is very common and serious problem, which causes considerable economic loss through out the world. Although it is inevitable but proper maintenance, good design and effective inhibitors may control it. Corrosion of mild steel and it alloys in different acid media has been extensively studied ${ }^{2-5}$. 
It has been established that the organic compounds having heteroatoms like $\mathrm{N}, \mathrm{O}$ and $\mathrm{S}$, which have lone pair of electrons, adsorb on the metal surface $e^{6-12}$ and thus cause inhibition.

Effects of $N$-and $S$-containing organic compounds such as substituted benzothizoles and various organic $S$-containing compounds of $\mathrm{Fe}$ and Mild steel have been studied ${ }^{13-15}$. In the present investigation the inhibition efficiency of four newly synthesized Schiff bases viz. $\mathrm{N}$ 4-(Diethylamino) salicylidine-2-amino-5-chloropyridine ( $\left.\mathrm{SB}_{1}\right), \quad N$-(4-ethylbenzalidine)-2amino-5-chloropyridine $\left(\mathrm{SB}_{2}\right), \mathrm{N}$ - (4-ethylbenzalidine)-2-amino-5-bromopyridine $\left(\mathrm{SB}_{3}\right), \mathrm{N}$ 4-(Diethylamino) salicylidine-2-amino-5-bromopyridine $\left(\mathrm{SB}_{4}\right)$, have been studied for mild steel in $\mathrm{HCl}$ and $\mathrm{H}_{2} \mathrm{SO}_{4}$ solutions.

\section{Experimental}

A rectangular specimen of mild steel having dimensions $2.0 \mathrm{~cm} \times 2.0 \mathrm{~cm} \times 0.021 \mathrm{~cm}$ containing a small hole of about $0.02 \mathrm{~cm}$ diameter near the upper edge were taken. The approximate composition of the specimen was $99.7 \% \mathrm{Fe}, 0.11 \% \mathrm{C}, 0.5 \% \mathrm{Mg}, 0.13 \% \mathrm{Si}$, $0.06 \%$ S. Specimens were cut from a sheet and thoroughly cleaned by buffing to produce a spotless finish and then degreased. Finally each specimen was washed with acetone and dried. All chemicals used for the synthesis of Schiff bases were of analytical reagent grade and solutions of $\mathrm{HCl}$ and $\mathrm{H}_{2} \mathrm{SO}_{4}$ were prepared in double distilled $\mathrm{H}_{2} \mathrm{O}$. All the Schiff bases were prepared by conventional method i.e. by refluxing equimolar quantities of respective aldehydes and amines. Each specimen was weighed accurately with a digital balance up to the accuracy of $0.1 \mathrm{mg}$ and then suspended in a borosilicate glass beaker of $50 \mathrm{~mL}$ capacity containing test solution, by a $V$-shaped glass hook made by capillary tubes at room temperature. After the test, Specimens were cleaned with running $\mathrm{H}_{2} \mathrm{O}$ and dried with hot air drier and then weighed again. The Percentage inhibition efficiency $(\eta \%)$ was calculated as ${ }^{16}$.

$$
\eta \%=\frac{\left(\Delta M_{u}-\Delta M_{i}\right)}{\Delta M_{u}} \times 100
$$

Where $\Delta \mathrm{M}_{\mathrm{u}}=$ Mass loss of specimen in uninhibited solution, $\Delta \mathrm{M}_{\mathrm{i}}=$ Mass loss of specimen in inhibited solution. The corrosion rate in $\mathrm{mm} / \mathrm{yr}$ (milli mils per year) can be obtained by following equations ${ }^{17}$.

$$
\text { C.R }(\mathrm{mm} / \mathrm{yr})=\frac{\Delta M \times 87.6}{A T D}
$$

$\Delta \mathrm{M}=$ Mass loss in $\mathrm{mg}, \mathrm{A}=$ Exposed area of metal surface in $\mathrm{cm}^{2}, \mathrm{~T}=$ Time of exposure in hours, $\mathrm{D}=$ Metal density in $\mathrm{gm} / \mathrm{cm}^{3}$ and $1 \mathrm{mil}=0.001$ inch. Surface coverage $(\theta)$ of metal specimen by inhibitor was calculated as

$$
\theta=\frac{\left(\Delta M_{u}-\Delta M_{i}\right)}{\Delta M_{u}}
$$

Where $\Delta \mathrm{M}_{\mathrm{u}}$ and $\Delta \mathrm{M}_{\mathrm{i}}$ have same significances as given in the formula of inhibition efficiency $(\eta \%)$.

\section{Results and Discussion}

Mass loss $(\Delta \mathrm{M})$ and percentage inhibition efficiencies $(\eta \%)$ for different concentrations of $\mathrm{HCl}$ and inhibitors are shown in Table 1 and those of for the $\mathrm{H}_{2} \mathrm{SO}_{4}$ are shown in Table 2. It is observed that percentage inhibition efficiency $(\eta \%)$ increases with increase in the concentrations of both the acids and also with the increase in the concentration of inhibitors. 
Table 1. Mass loss $(\Delta \mathrm{M})$ \& inhibition efficiency $(\eta \%)$ for mild steel in $\mathrm{HCl}$ solution with given inhibitor additions; Temperature: $-30 \pm 0.1{ }^{\circ} \mathrm{C}$

\begin{tabular}{|c|c|c|c|c|c|c|c|c|}
\hline \multirow{2}{*}{$\begin{array}{c}\text { Inhibitor } \\
\text { Concentration, } \%\end{array}$} & \multicolumn{2}{|c|}{$\mathrm{N} \mathrm{HCl}, 88 \mathrm{~h}$} & \multicolumn{2}{|c|}{$0.5 \mathrm{~N} \mathrm{HCl}, 72 \mathrm{~h}$} & \multicolumn{2}{|c|}{$10 \mathrm{~N} \mathrm{HCl}, 64 \mathrm{~h}$} & \multicolumn{2}{|c|}{$10 \mathrm{~N} \mathrm{HCl}, 49 \mathrm{~h}$} \\
\hline & $\overline{\Delta \mathrm{M}, \mathrm{mg}}$ & $\eta \%$ & $\Delta \mathrm{M}, \mathrm{mg}$ & $\eta \%$ & $\Delta \mathrm{M}, \mathrm{mg}$ & $\eta \%$ & $\Delta \mathrm{M}, \mathrm{mg}$ & $\eta \%$ \\
\hline $\begin{array}{c}\text { Uninhibited } \\
\mathrm{SB}_{1}\end{array}$ & 21.3 & - & 37.9 & - & 43.4 & - & 153.6 & - \\
\hline 0.1 & 10.0 & 53.05 & 15.2 & 59.89 & 13.4 & 69.12 & 35.7 & 76.75 \\
\hline 0.3 & 7.3 & 65.72 & 12.4 & 67.28 & 10.5 & 75.80 & 28.5 & 81.44 \\
\hline 0.5 & 6.1 & 71.36 & 10.2 & 73.08 & 8.5 & 80.41 & 25.0 & 83.72 \\
\hline 0.7 & 5.2 & 75.58 & 8.4 & 77.83 & 5.6 & 87.09 & 14.3 & 90.69 \\
\hline $\mathrm{SB}_{2}$ & & & & & & & & \\
\hline 0.1 & 8.2 & 61.50 & 11.5 & 69.65 & 10.6 & 75.57 & 26.3 & 82.87 \\
\hline 0.3 & 6.8 & 68.07 & 9.8 & 74.14 & 8.8 & 79.72 & 19.0 & 87.63 \\
\hline 0.5 & 5.4 & 74.64 & 7.7 & 79.68 & 6.6 & 84.79 & 12.6 & 91.79 \\
\hline 0.7 & 3.8 & 82.15 & 5.3 & 86.01 & 4.3 & 90.09 & 6.5 & 95.76 \\
\hline $\mathrm{SB}_{3}$ & & & & & & & & \\
\hline 0.1 & 8.1 & 61.97 & 9.4 & 75.19 & 6.3 & 85.48 & 12.4 & 91.92 \\
\hline 0.3 & 6.9 & 67.60 & 7.9 & 79.15 & 4.9 & 88.70 & 8.1 & 94.72 \\
\hline 0.5 & 5.6 & 73.70 & 6.7 & 82.32 & 3.5 & 91.93 & 7.3 & 95.24 \\
\hline 0.7 & 3.8 & 82.15 & 4.3 & 88.65 & 2.5 & 94.23 & 3.5 & 97.72 \\
\hline $\mathrm{SB}_{4}$ & & & & & & & & \\
\hline 0.1 & 10.4 & 51.17 & 17.6 & 53.56 & 17.5 & 59.67 & 49.6 & 67.70 \\
\hline 0.3 & 9.0 & 57.74 & 15.4 & 59.36 & 15.8 & 63.59 & 42.3 & 72.46 \\
\hline 0.5 & 7.7 & 63.84 & 13.0 & 65.69 & 13.3 & 69.35 & 36.4 & 76.30 \\
\hline 0.7 & 6.3 & 70.42 & 10.8 & 71.50 & 11.7 & 73.04 & 27.6 & 82.03 \\
\hline
\end{tabular}

Table 2. Mass loss $\Delta \mathrm{M} \&$ inhibition efficiency $\eta \%$ for mild steel in $\mathrm{H}_{2} \mathrm{SO}_{4}$ solution with given inhibitor additions. Temperature: $-30 \pm 0.1{ }^{\circ} \mathrm{C}$

\begin{tabular}{cccccccccc}
\hline Inhibitor & \multicolumn{2}{c}{$0.1 \mathrm{~N} \mathrm{H}_{2} \mathrm{SO}_{4} 75 \mathrm{~h} 0.5 \mathrm{~N} \mathrm{H}_{2} \mathrm{SO}_{4}, 75 \mathrm{~h}$} & $1.0 \mathrm{~N} \mathrm{H}_{2} \mathrm{SO}_{4}, 75 \mathrm{~h} 2.0 \mathrm{~N} \mathrm{H}_{2} \mathrm{SO}_{4}, 75 \mathrm{~h}$ \\
\cline { 2 - 9 } Concentration, & $\Delta \mathrm{M}, \mathrm{mg}$ & $\eta \%$ & $\Delta \mathrm{M}, \mathrm{mg}$ & $\eta \%$ & $\Delta \mathrm{M}, \mathrm{mg}$ & $\eta \%$ & $\Delta \mathrm{M}, \mathrm{mg}$ & $\eta \%$ \\
\hline Uninhibited & 23.2 & - & 32.7 & - & 49.6 & - & 93.8 & - \\
$\mathrm{SB}_{1}$ & & & & & & & & \\
0.1 & 9.8 & 57.75 & 11.2 & 65.74 & 13.7 & 72.37 & 18.9 & 79.85 \\
0.3 & 7.3 & 68.53 & 10.4 & 68.19 & 10.6 & 78.62 & 14.7 & 84.32 \\
0.5 & 5.9 & 74.56 & 8.5 & 74.00 & 8.3 & 83.26 & 13.1 & 86.03 \\
0.7 & 4.6 & 80.17 & 6.4 & 80.42 & 4.8 & 90.32 & 6.2 & 93.39 \\
$\mathrm{SB}_{2}$ & & & & & & & & \\
0.1 & 8.7 & 62.50 & 8.8 & 73.08 & 9.2 & 81.45 & 11.2 & 88.05 \\
0.3 & 5.9 & 74.56 & 6.4 & 80.42 & 8.3 & 83.26 & 4.9 & 94.77 \\
0.5 & 5.1 & 78.01 & 5.7 & 82.56 & 4.8 & 90.32 & 4.5 & 95.20 \\
0.7 & 3.3 & 85.77 & 3.7 & 88.68 & 2.8 & 94.35 & 3.4 & 96.37 \\
$\mathrm{SB}_{3}$ & & & & & & & & \\
0.1 & 5.2 & 77.58 & 5.7 & 82.56 & 5.1 & 89.71 & 4.0 & 95.73 \\
0.3 & 4.3 & 81.46 & 4.6 & 85.93 & 3.7 & 92.54 & 2.7 & 97.12 \\
0.5 & 3.2 & 86.20 & 3.3 & 89.90 & 2.9 & 94.15 & 1.0 & 98.93 \\
0.7 & 2.3 & 90.08 & 1.9 & 94.18 & 2.0 & 95.96 & 0.2 & 99.78 \\
$\mathrm{SB}_{4}$ & & & & & & & & \\
0.1 & 9.7 & 58.18 & 12.9 & 60.55 & 16.0 & 67.74 & 25.7 & 72.60 \\
0.3 & 8.0 & 65.51 & 10.7 & 67.27 & 13.5 & 72.78 & 12.7 & 86.46 \\
0.5 & 7.1 & 69.39 & 9.6 & 70.64 & 11.6 & 76.61 & 9.5 & 89.87 \\
0.7 & 5.6 & 75.86 & 6.3 & 80.73 & 8.9 & 82.05 & 5.1 & 94.56 \\
\hline
\end{tabular}


All the four Schiff bases show maximum inhibition efficiency at the highest concentration of both the acids $2 \mathrm{~N}$ at their highest concentration i.e. $0.7 \%$. The maximum efficiency was shown by $\mathrm{N}$ - (4-ethylbenzalidine)-2-amino-5- bromopyridine $\left(\mathrm{SB}_{3}\right)$ in $\mathrm{HCl}(97.72 \%)$ and in $\mathrm{H}_{2} \mathrm{SO}_{4}(99.78 \%)$.

Corresponding corrosion rate $(\mathrm{mm} / \mathrm{yr})$ and surface coverage $(\theta)$ for $\mathrm{HCl}$ solutions are depicted in Table 3 where as for $\mathrm{H}_{2} \mathrm{SO}_{4}$ they are shown in Table 4. It is observed from both the tables that corrosion rate of mild steel decreases with the increase in the concentrations of inhibitors where as corrosion rate increases with the increase in the strength of $\mathrm{HCl}$ and $\mathrm{H}_{2} \mathrm{SO}_{4}$ solutions.

Corrosion rate of mild steel is much higher in $\mathrm{H}_{2} \mathrm{SO}_{4}$ than in $\mathrm{HCl}$. It means $\mathrm{H}_{2} \mathrm{SO}_{4}$ has more adverse effect on mild steel in comparison to $\mathrm{HCl}$. Values of surface coverage indicate that inhibitors are in general more effective in $\mathrm{H}_{2} \mathrm{SO}_{4}$ than in $\mathrm{HCl}$. Surface coverage $(\theta)$ of metal specimen by inhibitors increases with the increase in the acid strength as well as with the increase in the concentration of inhibitors. Maximum surface coverage is observed at the highest concentration $(2 \mathrm{~N})$ of acids at maximum concentration $(0.7 \%)$. Surface coverage $(\theta)$ and $\log [\theta / 1-\theta]$ values of mild steel in $\mathrm{HCl}$ solutions are depicted in Table 5. Where as, for $\mathrm{H}_{2} \mathrm{SO}_{4}$, these are shown in Table 6. It is observed from both the tables that as Surface coverage $(\theta)$ increases, the value of $\log [\theta / 1-\theta]$ also increases.

Table 3. Corrosion rate $(\mathrm{mm} / \mathrm{yr})$ \& surface coverage $(\theta)$ for mild steel in $\mathrm{HCl}$ solution with given inhibitor additions; Temperature: $-30 \pm 0.1{ }^{\circ} \mathrm{C}$; Effective area of specimen $=8 \mathrm{~cm}^{2}$

\begin{tabular}{|c|c|c|c|c|c|c|c|c|}
\hline \multirow{2}{*}{$\begin{array}{c}\text { Inhibitor } \\
\text { Concentration, } \\
\%\end{array}$} & \multicolumn{2}{|c|}{$\begin{array}{c}0.1 \mathrm{~N} \mathrm{HCl}, \\
88 \mathrm{~h}\end{array}$} & \multicolumn{2}{|c|}{$\begin{array}{c}0.5 \mathrm{~N} \mathrm{HCl}, \\
72 \mathrm{~h}\end{array}$} & \multicolumn{2}{|c|}{$\begin{array}{c}1.0 \mathrm{~N} \mathrm{HCl}, \\
64 \mathrm{~h}\end{array}$} & \multicolumn{2}{|c|}{$\begin{array}{c}2.0 \mathrm{~N} \mathrm{HCl}, \\
49 \mathrm{~h}\end{array}$} \\
\hline & $\begin{array}{c}\text { C.R } \\
(\mathrm{mm} / \mathrm{yr})\end{array}$ & $\begin{array}{l}\text { Surface } \\
\text { Cover. } \\
(\theta)\end{array}$ & $\begin{array}{c}\text { C.R } \\
(\mathrm{mm} / \mathrm{yr})\end{array}$ & $\begin{array}{l}\text { Surface } \\
\text { Cover. } \\
(\theta)\end{array}$ & $\begin{array}{c}\text { C.R } \\
(\mathrm{mm} / \mathrm{yr})\end{array}$ & $\begin{array}{c}\text { Surface } \\
\text { Cover. } \\
(\theta) \\
\end{array}$ & $\begin{array}{c}\text { C.R } \\
(\mathrm{mm} / \mathrm{yr})\end{array}$ & $\begin{array}{c}\text { Surface } \\
\text { Cover. } \\
(\theta) \\
\end{array}$ \\
\hline $\begin{array}{c}\text { Uninhibited } \\
\mathrm{SB}_{1}\end{array}$ & 0.39 & - & 0.84 & - & 10.85 & - & 5.01 & - \\
\hline 0.1 & 0.18 & 0.54 & 0.34 & 0.59 & 3.35 & 0.69 & 1.16 & 0.77 \\
\hline 0.3 & 0.13 & 0.66 & 0.27 & 0.68 & 2.62 & 0.76 & 0.93 & 0.81 \\
\hline 0.5 & 0.11 & 0.71 & 0.22 & 0.74 & 2.12 & 0.80 & 0.81 & 0.84 \\
\hline 0.7 & 0.09 & 0.76 & 0.18 & 0.78 & 1.40 & 0.87 & 0.46 & 0.91 \\
\hline $\mathrm{SB}_{2}$ & & & & & & & & \\
\hline 0.1 & 0.14 & 0.64 & 0.25 & 0.70 & 2.65 & 0.75 & 0.85 & 0.83 \\
\hline 0.3 & 0.12 & 0.69 & 0.21 & 0.74 & 2.20 & 0.79 & 0.62 & 0.87 \\
\hline 0.5 & 0.09 & 0.75 & 0.17 & 0.80 & 1.65 & 0.84 & 0.41 & 0.92 \\
\hline 0.7 & 0.06 & 0.82 & 0.12 & 0.86 & 1.07 & 0.90 & 0.21 & 0.96 \\
\hline $\mathrm{SB}_{3}$ & & & & & & & & \\
\hline 0.1 & 0.15 & 0.62 & 0.21 & 0.75 & 1.57 & 0.85 & 0.40 & 0.92 \\
\hline 0.3 & 0.12 & 0.68 & 0.17 & 0.79 & 1.22 & 0.88 & 0.26 & 0.94 \\
\hline 0.5 & 0.10 & 0.74 & 0.15 & 0.82 & 0.87 & 0.92 & 0.24 & 0.95 \\
\hline 0.7 & 0.07 & 0.82 & 0.10 & 0.89 & 0.62 & 0.94 & 0.11 & 0.98 \\
\hline $\mathrm{SB}_{4}$ & & & & & & & & \\
\hline 0.1 & 0.19 & 0.51 & 0.39 & 0.53 & 4.37 & 0.59 & 1.62 & 0.67 \\
\hline 0.3 & 0.16 & 0.58 & 0.34 & 0.59 & 3.95 & 0.63 & 1.38 & 0.72 \\
\hline 0.5 & 0.14 & 0.64 & 0.29 & 0.66 & 3.32 & 0.69 & 1.18 & 0.76 \\
\hline 0.7 & 0.11 & 0.70 & 0.24 & 0.71 & 2.92 & 0.73 & 0.90 & 0.82 \\
\hline
\end{tabular}


Table 4. Corrosion rate $(\mathrm{mm} / \mathrm{yr})$ and surface coverage $(\theta)$ for mild steel in $\mathrm{H}_{2} \mathrm{SO}_{4}$ solution with given inhibitor additions; Temperature: $-30+0.1^{\circ} \mathrm{C}$, Effective area of specimen $=8 \mathrm{~cm}^{2}$

\begin{tabular}{|c|c|c|c|c|c|c|c|c|}
\hline \multirow{2}{*}{$\begin{array}{c}\text { Inhibitor } \\
\text { Concentration, } \%\end{array}$} & \multicolumn{4}{|c|}{$0.1 \mathrm{~N} \mathrm{H}_{2} \mathrm{SO}_{4}, 75 \mathrm{~h} 0.5 \mathrm{~N} \mathrm{H}_{2} \mathrm{SO}_{4}, 75 \mathrm{~h}$} & \multicolumn{4}{|c|}{$1.0 \mathrm{~N} \mathrm{H}_{2} \mathrm{SO}_{4}, 75 \mathrm{~h} 2.0 \mathrm{~N} \mathrm{H}_{2} \mathrm{SO}_{4}, 75 \mathrm{~h}$} \\
\hline & $\begin{array}{c}\mathrm{C} . \mathrm{R} \\
(\mathrm{mmpy})\end{array}$ & $\begin{array}{c}\text { Surface } \\
\text { Cover. }(\theta)\end{array}$ & $\begin{array}{c}\text { C.R } \\
\text { (mmpy) }\end{array}$ & $\begin{array}{c}\text { Surface } \\
\text { Cover. }(\theta) \\
\end{array}$ & $\begin{array}{c}\text { C.R } \\
\text { (mmpy) }\end{array}$ & $\begin{array}{c}\text { Surface } \\
\text { Cover. }(\theta) \\
\end{array}$ & $\begin{array}{c}\text { C.R } \\
\text { (mmpy) }\end{array}$ & $\begin{array}{c}\text { Surface } \\
\text { Cover. }(\theta) \\
\end{array}$ \\
\hline $\begin{array}{c}\text { Uninhibited } \\
\mathrm{SB}_{1}\end{array}$ & 0.50 & - & 0.70 & - & 1.06 & - & 2.00 & - \\
\hline 0.1 & 0.21 & 0.58 & 0.24 & 0.65 & 0.29 & 0.72 & 0.40 & 0.80 \\
\hline 0.3 & 0.15 & 0.68 & 0.22 & 0.68 & 0.22 & 0.79 & 0.31 & 0.84 \\
\hline 0.5 & 0.12 & 0.74 & 0.18 & 0.74 & 0.18 & 0.83 & 0.28 & 0.86 \\
\hline 0.7 & 0.09 & 0.80 & 0.13 & 0.80 & 0.10 & 0.90 & 0.13 & 0.93 \\
\hline $\mathrm{SB}_{2}$ & & & & & & & & \\
\hline 0.1 & 0.18 & 0.62 & 0.19 & 0.73 & 0.20 & 0.81 & 0.24 & 0.88 \\
\hline 0.3 & 0.12 & 0.75 & 0.13 & 0.80 & 0.17 & 0.83 & 0.10 & 0.95 \\
\hline 0.5 & 0.10 & 0.78 & 0.12 & 0.83 & 0.10 & 0.90 & 0.09 & 0.95 \\
\hline 0.7 & 0.07 & 0.86 & 0.08 & 0.89 & 0.06 & 0.94 & 0.07 & 0.96 \\
\hline $\mathrm{SB}_{3}$ & & & & & & & & \\
\hline 0.1 & 0.11 & 0.78 & 0.12 & 0.82 & 0.10 & 0.90 & 0.08 & 0.96 \\
\hline 0.3 & 0.09 & 0.81 & 0.09 & 0.86 & 0.07 & 0.92 & 0.05 & 0.97 \\
\hline 0.5 & 0.06 & 0.86 & 0.07 & 0.90 & 0.06 & 0.94 & 0.02 & 0.98 \\
\hline 0.7 & 0.04 & 0.90 & 0.04 & 0.94 & 0.04 & 0.96 & 0.004 & 0.99 \\
\hline $\mathrm{SB}_{4}$ & & & & & & & & \\
\hline 0.1 & 0.20 & 0.58 & 0.27 & 0.61 & 0.34 & 0.67 & 0.55 & 0.72 \\
\hline 0.3 & 0.17 & 0.65 & 0.29 & 0.68 & 0.28 & 0.73 & 0.27 & 0.86 \\
\hline 0.5 & 0.15 & 0.69 & 0.20 & 0.71 & 0.25 & 0.77 & 0.20 & 0.90 \\
\hline 0.7 & 0.12 & 0.76 & 0.13 & 0.81 & 0.19 & 0.82 & 0.11 & 0.94 \\
\hline
\end{tabular}

Table 5. Surface coverage $(\theta)$ and $\log \left[\frac{\theta}{1-\theta}\right]$ for mild steel in $\mathrm{HCl}$ solutions with given inhibitor additions Effective area of specimen $=8 \mathrm{~cm}^{2}$

\begin{tabular}{|c|c|c|c|c|c|c|c|c|}
\hline \multirow{2}{*}{ 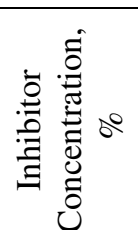 } & \multicolumn{2}{|c|}{$\begin{array}{c}0.1 \mathrm{NHCl} \\
(88 \mathrm{~h})\end{array}$} & \multicolumn{2}{|c|}{$\begin{array}{c}0.5 \mathrm{~N} \mathrm{HCl} \\
(72 \mathrm{~h})\end{array}$} & \multicolumn{2}{|c|}{$\begin{array}{c}1.0 \mathrm{~N} \mathrm{HCl} \\
(64 \mathrm{~h})\end{array}$} & \multicolumn{2}{|c|}{$\begin{array}{c}2.0 \mathrm{~N} \mathrm{HCl} \\
(49 \mathrm{~h})\end{array}$} \\
\hline & $\begin{array}{c}\text { Surface } \\
\text { coverage } \\
(\theta)\end{array}$ & $\begin{array}{c}\log \\
{\left[\frac{\theta}{1-\theta}\right]}\end{array}$ & $\begin{array}{c}\text { Surface } \\
\text { coverage } \\
(\theta)\end{array}$ & $\begin{array}{l}\log \\
{\left[\frac{\theta}{1-\theta}\right]}\end{array}$ & $\begin{array}{c}\text { Surface } \\
\text { coverage } \\
(\theta)\end{array}$ & $\begin{array}{c}\log \\
{\left[\frac{\theta}{1-\theta}\right]}\end{array}$ & $\begin{array}{c}\text { Surface } \\
\text { coverage } \\
(\theta)\end{array}$ & $\begin{array}{l}\log \\
{\left[\frac{\theta}{1-\theta}\right.}\end{array}$ \\
\hline
\end{tabular}

\begin{tabular}{ccccccccc}
\hline Uninhibited & - & - & - & - & - & - & - & - \\
$\mathrm{SB}_{1}$ & & & & & & & & \\
0.1 & 0.53 & 0.53 & 0.60 & 0.17 & 0.70 & 0.35 & 0.77 & .0 .52 \\
0.3 & 0.66 & 0.28 & 0.67 & 0.31 & 0.76 & 0.49 & 0.81 & 0.64 \\
0.5 & 0.71 & 0.40 & 0.73 & 0.43 & 0.80 & 0.61 & 0.84 & 0.71 \\
0.7 & 0.75 & 0.49 & 0.78 & 0.54 & 0.87 & 0.83 & 0.90 & 0.99 \\
$\mathrm{SB}_{2}$ & & & & & & & & \\
0.1 & 0.61 & 0.20 & 0.70 & 0.36 & 0.75 & 0.50 & 0.83 & 0.68 \\
0.3 & 0.68 & 0.33 & 0.74 & 0.46 & 0.80 & 0.60 & 0.88 & 0.85 \\
0.5 & 0.75 & 0.47 & 0.80 & 0.60 & 0.85 & 0.75 & 0.92 & 1.05 \\
0.7 & 0.82 & 0.66 & 0.86 & 0.79 & 0.90 & 0.96 & 0.96 & 1.35 \\
\hline & & & & & & & & Contd..
\end{tabular}




\begin{tabular}{ccccccccc}
$\mathrm{SB}_{3}$ & & & & & & & & \\
0.1 & 0.62 & 0.21 & 0.75 & 0.48 & 0.85 & 0.77 & 0.92 & 1.05 \\
0.3 & 0.68 & 0.32 & 0.79 & 0.58 & 0.89 & 0.89 & 0.95 & 1.25 \\
0.5 & 0.74 & 0.45 & 0.82 & 0.67 & 0.92 & 1.06 & 0.95 & 1.30 \\
0.7 & 0.82 & 0.66 & 0.89 & 0.89 & 0.94 & 1.21 & 0.98 & 1.63 \\
$\mathrm{SB}_{4}$ & & & & & & & & \\
0.1 & 0.50 & -0.01 & 0.53 & 0.062 & 0.60 & 0.17 & 0.68 & 0.32 \\
0.3 & 0.58 & 0.13 & 0.59 & 0.16 & 0.63 & 0.24 & 0.72 & 0.42 \\
0.5 & 0.64 & 0.25 & 0.66 & 0.28 & 0.69 & 0.35 & 0.76 & 0.50 \\
0.7 & 0.70 & 0.38 & 0.71 & 0.40 & 0.73 & 0.43 & 0.82 & 0.66 \\
\hline
\end{tabular}

Table 6. Surface coverage $(\theta)$ and $\log \left[\frac{\theta}{1-\theta}\right]$ for mild steel in $\mathrm{H}_{2} \mathrm{SO}_{4}$ solutions with given inhibitor additions. Effective area of specimen $=8 \mathrm{~cm}^{2}$

\begin{tabular}{|c|c|c|c|c|c|c|c|c|}
\hline \multirow{2}{*}{ 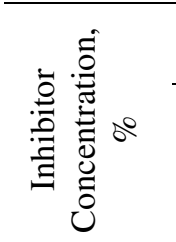 } & \multicolumn{2}{|c|}{$\begin{array}{c}0.1 \mathrm{~N} \mathrm{H}_{2} \mathrm{SO}_{4} \\
(75 \mathrm{~h})\end{array}$} & \multicolumn{2}{|c|}{$\begin{array}{c}0.5 \mathrm{~N} \mathrm{H}_{2} \mathrm{SO}_{4} \\
(75 \mathrm{~h})\end{array}$} & \multicolumn{2}{|c|}{$\begin{array}{c}1.0 \mathrm{~N} \mathrm{H}_{2} \mathrm{SO}_{4} \\
(75 \mathrm{~h})\end{array}$} & \multicolumn{2}{|c|}{$\begin{array}{c}2.0 \mathrm{~N} \mathrm{H}_{2} \mathrm{SO}_{4} \\
(75 \mathrm{~h})\end{array}$} \\
\hline & $\begin{array}{c}\text { Surface } \\
\text { coverage } \\
(\theta)\end{array}$ & {$\left[\frac{\theta}{1-\theta}\right.$} & $\begin{array}{c}\text { Surface } \\
\text { coverage } \\
(\theta)\end{array}$ & {$\left[\begin{array}{c}\log \\
\frac{\theta}{1-\theta}\end{array}\right.$} & $\begin{array}{c}\text { Surface } \\
\text { coverage } \\
(\theta)\end{array}$ & $\begin{array}{c}\log \\
{\left[\frac{\theta}{1-\theta}\right]}\end{array}$ & $\begin{array}{c}\text { Surface } \\
\text { coverage } \\
(\theta)\end{array}$ & $\begin{array}{c}\log \\
{\left[\frac{\theta}{1-\theta}\right]}\end{array}$ \\
\hline $\begin{array}{c}\text { Uninhibited } \\
\mathrm{SB}_{1}\end{array}$ & - & - & - & - & - & - & - & - \\
\hline 0.1 & 0.58 & 0.13 & 0.66 & 0.28 & 0.72 & 0.42 & 0.80 & 0.60 \\
\hline 0.3 & 0.68 & 0.34 & 0.68 & 0.33 & 0.79 & 0.56 & 0.84 & 0.73 \\
\hline 0.5 & 0.74 & 0.47 & 0.74 & 0.45 & 0.83 & 0.70 & 0.86 & 0.79 \\
\hline 0.7 & 0.80 & 0.60 & 0.80 & 0.61 & 0.90 & 1.10 & 0.93 & 1.15 \\
\hline $\mathrm{SB}_{2}$ & & & & & & & & \\
\hline 0.1 & 0.62 & 0.22 & 0.73 & 0.43 & 0.81 & 0.64 & 0.88 & 0.87 \\
\hline 0.3 & 0.74 & 0.47 & 0.80 & 0.61 & 0.83 & 0.70 & 0.95 & 1.26 \\
\hline 0.5 & 0.78 & 0.55 & 0.82 & 0.67 & 0.90 & 0.97 & 0.95 & 1.30 \\
\hline 0.7 & 0.86 & 0.78 & 0.89 & 0.89 & 0.94 & 1.22 & 0.96 & 1.42 \\
\hline $\mathrm{SB}_{3}$ & & & & & & & & \\
\hline 0.1 & 0.77 & 0.54 & 0.82 & 0.67 & 090 & 0.94 & 0.96 & 1.35 \\
\hline 0.3 & 0.81 & 0.64 & 0.86 & 0.78 & 0.92 & 1.09 & 0.97 & 1.53 \\
\hline 0.5 & 0.86 & 0.79 & 0.89 & 0.94 & 0.94 & 1.20 & 0.99 & 1.96 \\
\hline 0.7 & 0.90 & 0.96 & 0.94 & 1.20 & 0.96 & 1.37 & 1.09 & 2.66 \\
\hline $\mathrm{SB}_{4}$ & & & & & & & & \\
\hline 0.1 & 0.58 & 0.14 & 0.60 & 0.19 & 0.68 & 0.32 & 0.73 & 0.42 \\
\hline 0.3 & 0.65 & 0.28 & 0.67 & 0.31 & 0.73 & 0.43 & 0.86 & 0.80 \\
\hline 0.5 & 0.69 & 0.35 & 0.70 & 0.38 & 0.77 & 0.51 & 0.89 & 0.95 \\
\hline 0.7 & 0.76 & 0.49 & 0.80 & 0.62 & 0.82 & 0.66 & 0.94 & 1.24 \\
\hline
\end{tabular}

Generally the organic molecules containing heteroatoms like oxygen, sulphur and nitrogen cause blockage of active sites on the metallic surface, thus resulting in the decrease in corrosion rate. Nitrogen atom present in Schiff bases has lone pair of electrons and thus Schiff base forms a monolayer on the metallic surface. The presence of $-\mathrm{C}_{2} \mathrm{H}_{5}$ and $-\mathrm{OH}$ groups in Schiff bases further increases the electron density and thus increases the inhibition efficiency of inhibitor. It has been observed that inhibition efficiency is higher in higher concentrations of acids. This may be due to the fact that in strong acidic conditions ionization of Schiff base 
increases which favours the adsorption strongly and thus further reduces the exposed area of metal which results further increase in inhibition efficiency.

Langmuir adsorption isotherm plot (graph between $\log C$ and $\log [\theta / 1-\theta]$ for mild steel in $0.1 \mathrm{~N} \mathrm{HCl}$ and in $0.1 \mathrm{~N} \mathrm{H}_{2} \mathrm{SO}_{4}$ containing the inhibitors as Schiff Bases are shown in Figure 1 and 2.

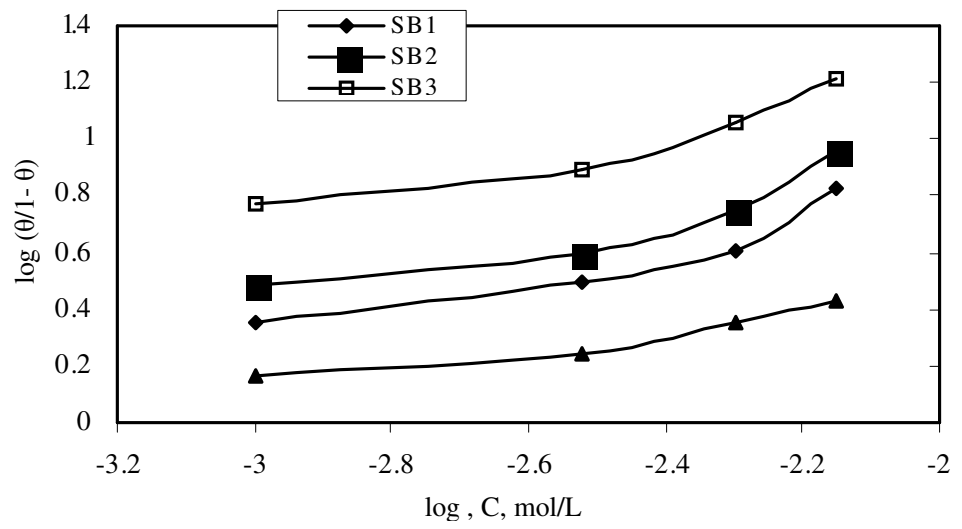

Figure 1. Langmuir adsorption isotherms for mild steel in $1.0 \mathrm{~N} \mathrm{HCl}$

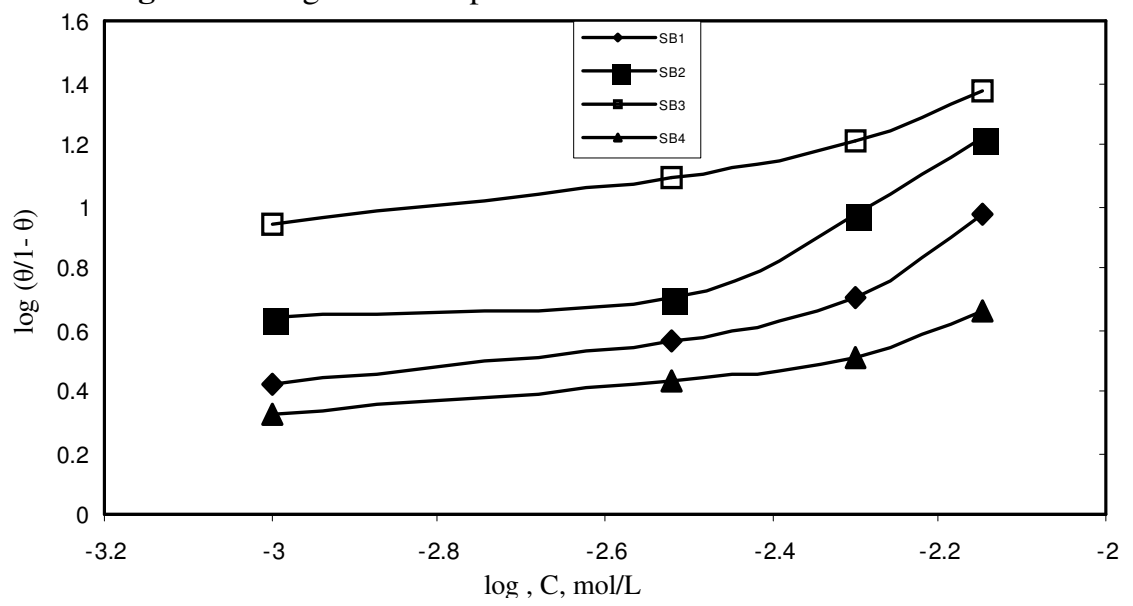

Figure 2. Langmuir adsorption isotherms for mild steel in $1.0 \mathrm{~N} \mathrm{H}_{2} \mathrm{SO}_{4}$

\section{Conclusion}

A study of five synthesized bases has shown them effective corrosion inhibitors for mild steel in $\mathrm{HCl}$ and $\mathrm{H}_{2} \mathrm{SO}_{4}$ acid solutions. Mass loss method has shown that efficiencies of inhibitors increase with increase in the concentration of acids as well as those of inhibitors. Among the four Schiff bases under study maximum inhibition efficiency was shown by $\mathrm{SB}_{3}$ in both acid solutions at $0.7 \%$ concentrations at the highest $(2 \mathrm{~N})$ strength.

It has been observed that corrosion rate of mild steel is much higher in $\mathrm{H}_{2} \mathrm{SO}_{4}$ than in $\mathrm{HCl}$ which gives the conclusion that $\mathrm{H}_{2} \mathrm{SO}_{4}$ is much more corrosive for mild steel than $\mathrm{HCl}$. Results of surface coverage indicate that synthesized Schiff bases are more effective in $\mathrm{H}_{2} \mathrm{SO}_{4}$ than in $\mathrm{HCl}$. 


\section{References}

1. Achouri M, Kertit S, Salem M, Essassi E M and Jellal M, Bull Electrochem., 1998, 14(12), 462.

2. Allah A G G and Tamous H M, Bull Electrochem., 1995, 11, 178.

3. Bastidas J M, Damborenea J De and Lazquez A J, J Appl Electrochem., 1997, 27, 345.

4. Schweinsberg D P and Ashworth V, Corros Sci., 1988, 28, 539.

5. Denny A Jones, Principles and Prevention of Corrosion, $2^{\text {nd }}$ Ed., Prentice Hall, Upper Saddle River, NJ 1996, p.34.

6. $\quad$ Stupnisek-Lisac E and Podbrscek S, J Appl Elecrochem., 1994, 24, 779.

7. Reverdin F, Helv Chim Acta., 1927, 10, 34.

8. Talati J D and Gandhi D K, Indian J Technol., 1991, 29, 277.

9. Laskawiec J, Sozanska M, Trzcionka B and Sukurczynska J, Koroz, 1995, 38, 249.

10. Stupnisek-Lisac E and Metikos-Hukovic M, Br Corros. J., 1993, 28, 74.

11. Quarishi M A, Rawat J and Ajmal M, Corrosion and its Control, Proc Int Conf Corros., 1997, 2, 634.

12. Moretti G, Quartarone G, Tassar A and Zingales A, Electrochem Acta., 1996, 41, 1971.

13. Shibad P R and Adhe K N, J Electrochem Soc (India), 1981, 30, 103.

14. Quraishi M A, Khan M A W, Jamal D and Ajmal M, Trans Indian Inst Met., 1998, 51, 431.

15. Rajendaran S, Apparo B V and Palaniswamy N, Anti Corros Methods Mater., 2000, 47, 294.

16. Raicheva S N, Aleksivev B V and Sokolova E I, Corros Sci., 1993, 34, 343.

17. Shalaby M N, Osman M M and ElFeky A A, Anti Corros Methods Mater., 1999, 46, 254. 


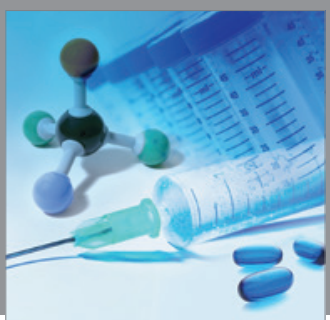

International Journal of

Medicinal Chemistry

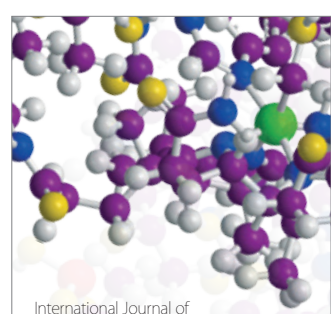

Carbohydrate Chemistry

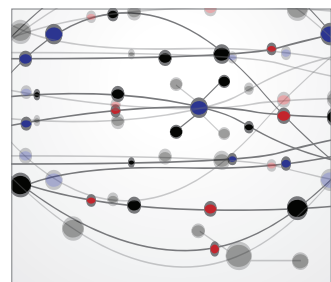

The Scientific World Journal
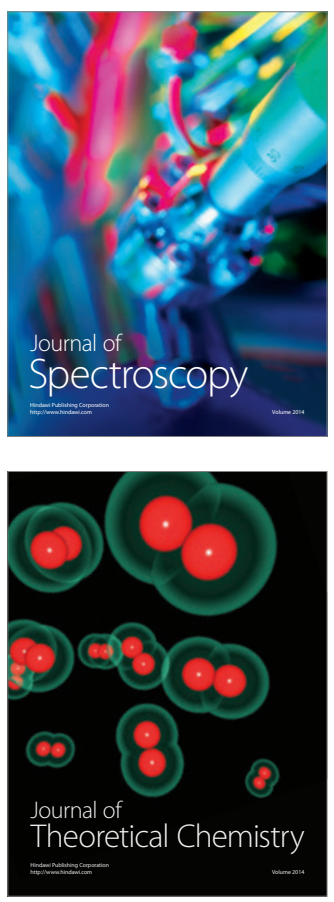
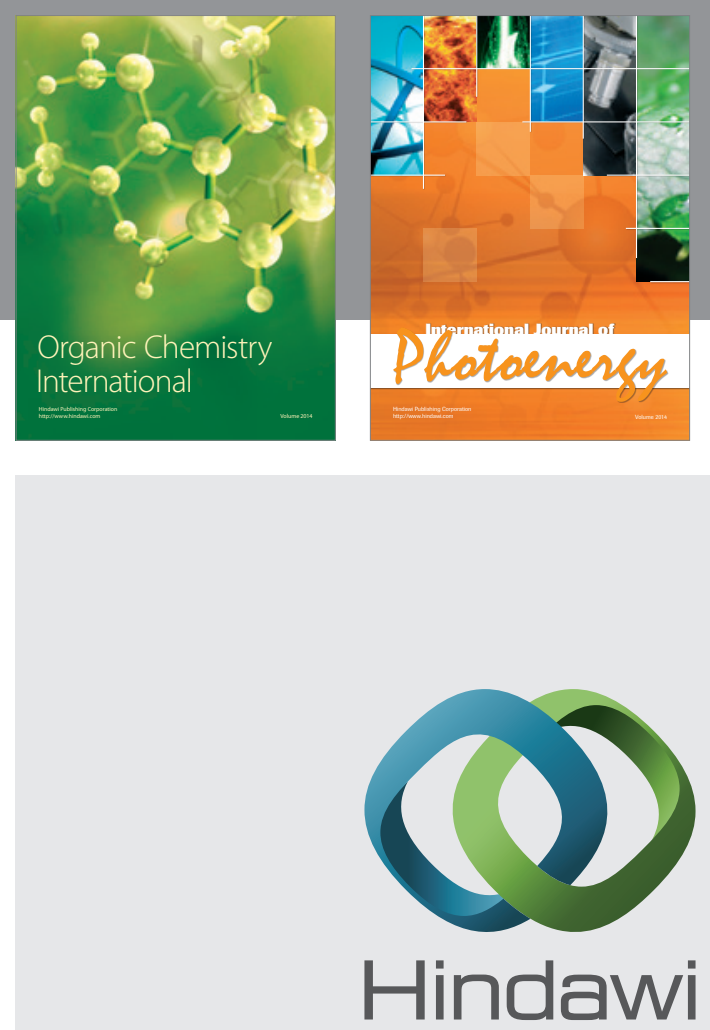

Submit your manuscripts at

http://www.hindawi.com
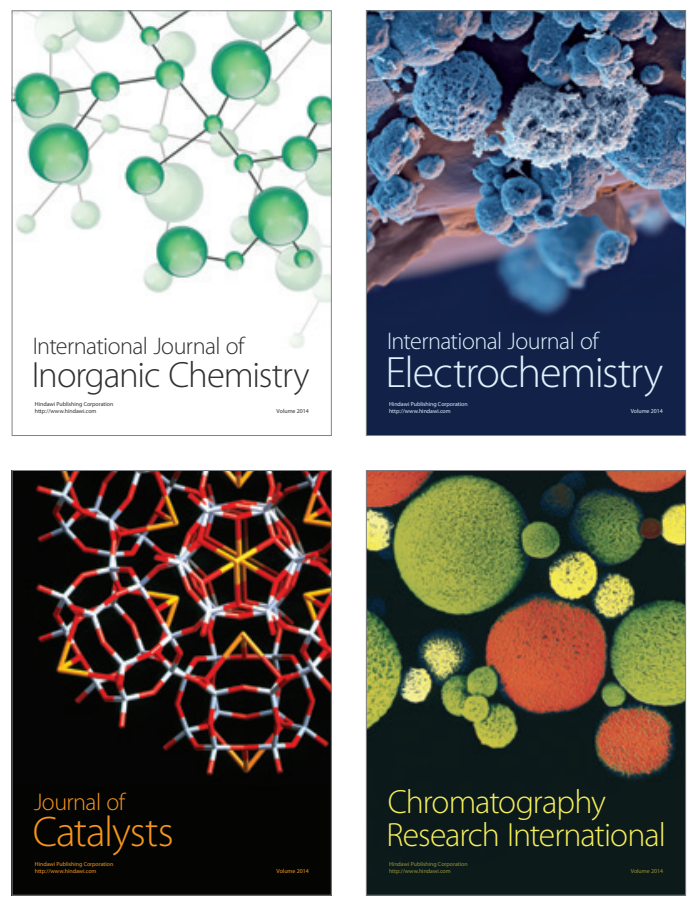
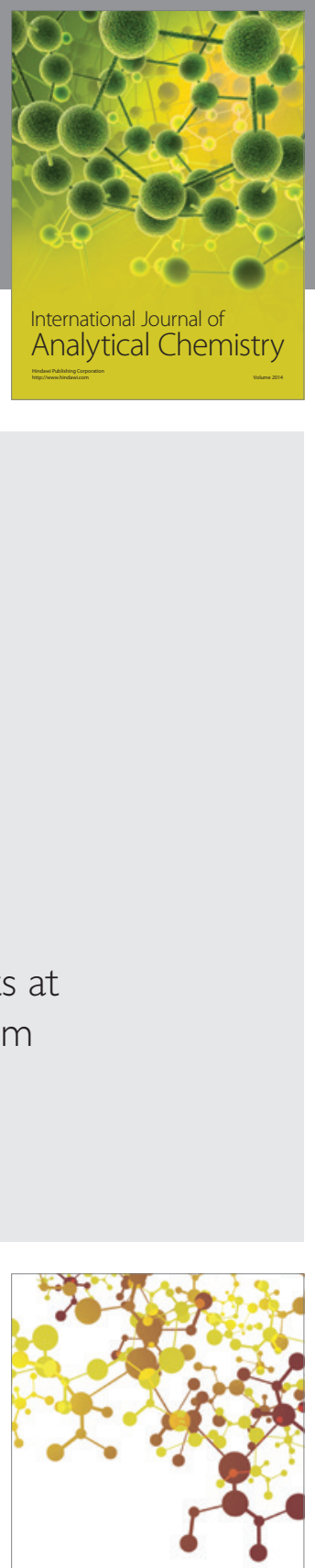

Journal of

Applied Chemistry
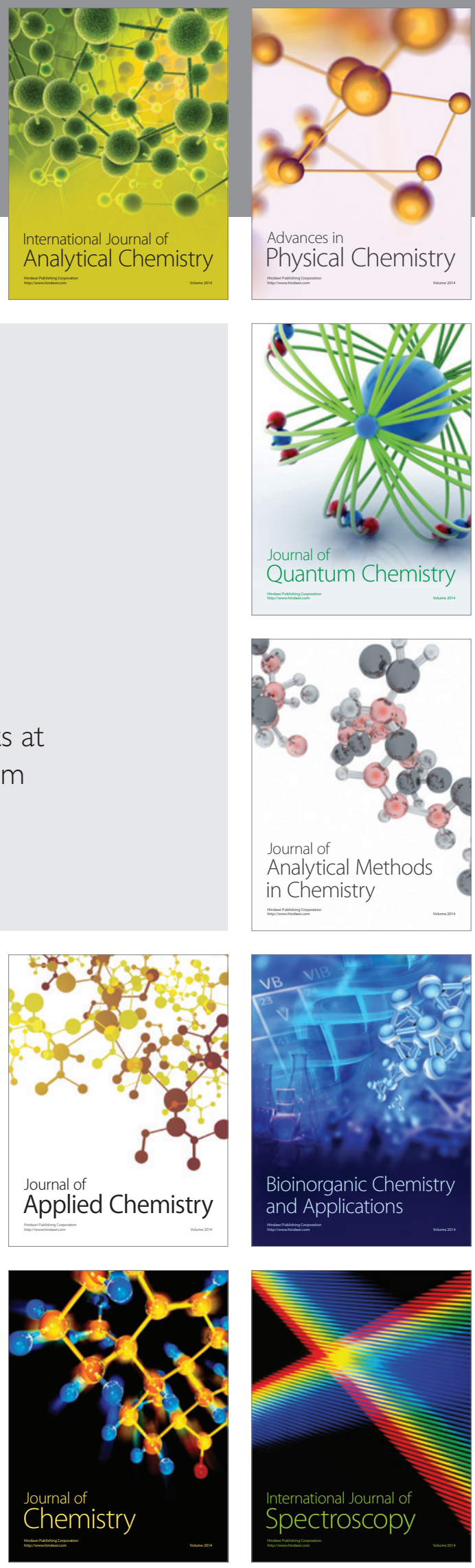